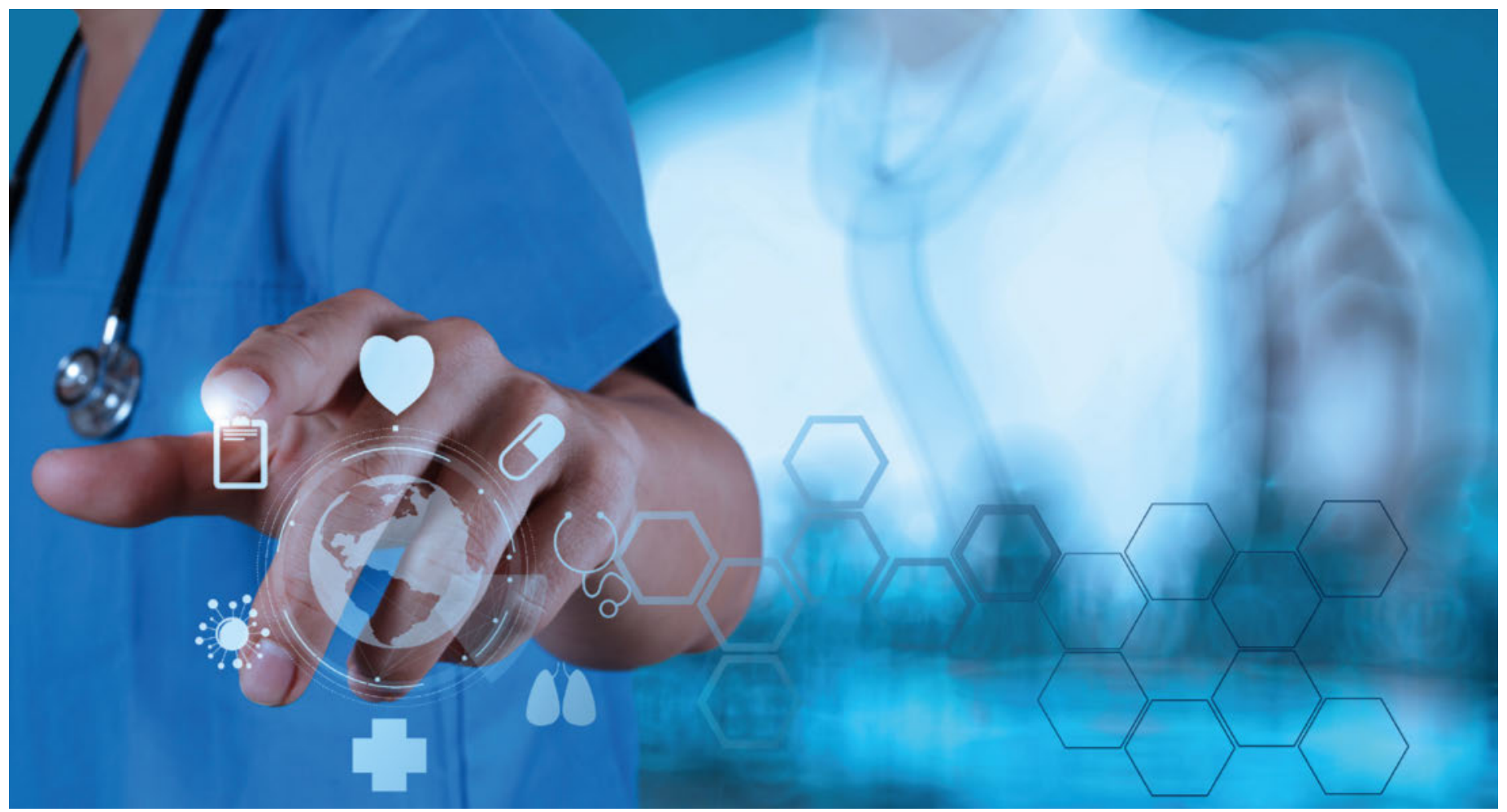

\title{
Medizin am Wendepunkt - Medizin am Ende. Punkt?
}

\section{Bernhard Gurtner}

In letzter Zeit haben Cyberkriminelle mit rasch ansteigenden Opferzahlen die Computer und Netzwerke unvorsichtiger Spitäler und Arztpraxen mit Sperrtrojanern (Ransomware) verschlüsselt und nur gegen Lösegeld wieder freigegeben. Pascal Lamis, Leiter der Meldestelle MELANI des Bundes, hat die Problematik und Sicherheitstipps in einem kurzen ersten Beitrag zur Artikelserie «FMH eHealth» leicht verständlich dargelegt [1].

Auch die NZZ am Sonntag hat am 5. Februar 2017 einen alarmierenden Bericht von Martin Linder veröffentlicht. Längst sei das Hacking von Spitälern weltweit ein lukratives Geschäft, über das Betroffene lieber schweigen, um sich nicht zu blamieren. Hacker können nicht nur gespeicherte Daten blockieren, sondern auch die

\section{Google DeepMind konnte 2016 mit drei Londoner Spitälern des Royal Free NHS Trust eine Datenübermittlung vereinbaren.}

Software von Labor-, Röntgen- oder IPS-Geräten mit Schadprogrammen infizieren. Über WLAN und Inter- net ferngesteuerte Herzschrittmacher oder Insulinpumpen sind ebenfalls nicht geschützt.

Vor diesem Hintergrund erstaunt die Euphorie der BigData-Protagonisten, welche die Software einer künst-

\section{Datengestütztes Qualitätsmanagement ist auch in der Schweiz ausgelagert worden.}

lichen Intelligenz freiwillig mit immer mehr Daten füttern wollen, die diese aus allen Bereichen des $\mathrm{Ge}$ sundheitswesens absaugt und angeblich zugriffsicher einlagert und verarbeitet. Adrian Ritter hat seinen SÄZ-Bericht über eine diesbezügliche Tagung an der Universität Zürich mit wohlberechtigten Fragen eingeleitet: Stellen in Zukunft Algorithmen statt Ärzte Diagnosen? Werden digitale Daten das medizinische Personal administrativ entlasten [2]?

DeepMind ist ein 2014 von Google mit etwa 500 Millionen Dollar aufgekauftes britisches Start-up-Unternehmen, das sich in der Entwicklung künstlicher Intelligenz spezialisiert hat. Google DeepMind konnte 2016 mit drei Londoner Spitälern des Royal Free NHS Trust 
eine Datenübermittlung vereinbaren, welche nicht nur die Krankengeschichten von 1,6 Millionen NHSVersicherten und alle ihre Befunde umfasst, sondern sogar auch die Zimmernummern und Besucher der Hospitalisierten [3] ... Wer? Wann? Orwell 1984?

Dank dieser im ersten Versuchsjahr noch unentgeltlichen Datensammlung verspricht die tiefsinnige Firma hochstaplerisch unglaubliche Verbesserungen: 500000 administrative Arbeitsstunden würden eingespart für vermehrte Patientenkontakte (viel eher wohl für Pflegestellenabbau). Die integrierte iPhone-App «Streams» warne frühzeitig vor Nierenversagen oder anderen Gefahren, welche die Ärzte im gewaltigen Datenstrom übersehen haben. So seien jährlich 10000 Todesfälle zu vermeiden. Potztausend, welche Oligurien und Kreatininwerte haben denn die Kollegen bisher verbummelt?

Datengestütztes Qualitätsmanagement ist auch in der Schweiz ausgelagert worden. Der Verein Outcome, der nach einem kostspieligen Probelauf in Zürich die öffentlichen Spitäler einiger Kantone mit Papierlawinen von Fragebogen eindecken durfte, fusionierte $2011 \mathrm{mit}$ der Health Care Research Institute AG (hcri), deren Softwarelösung Q1 die Plattform für zahlreiche Kunden aus

\section{Die Selbstmessung der Blutdruck- oder Blutzuckerwerte hat vielen zu besserer Kontrolle verholfen, aber auch ängstliche Personen verunsichert.}

dem Gesundheitsbereich bietet. Im Oktober 2015 wurde hcri ihrerseits von Die Post übernommen. Deren E-Health-Plattform vivates soll zur Schweizer Standardlösung werden. Die Post will mit verbesserten und übergreifenden Gesundheitsprozessen das Kostenwachstum begrenzen und das Gesundheitswesen für alle Marktteilnehmer effizienter und transparenter machen. Dabei steht das Wohl der Patienten immer im Mittelpunkt (Medienmitteilung vom 20.10.2015). Die POST als Gemischtwarenhandlung für Briefmarken, Papeteriebedarf, Schleckereien, Versicherungsabschlüsse und Gesundheitsdatenverwaltung?

Der kalifornische Datamaniker David B. Agus hat 2012 in seinem Bestseller The End of Illness dank recht- zeitiger Prävention angekündigt: "Routine blood tests will involve thousands of measurements to test for various diseases and genetic predispositions.» Sein Personal Genetic Profile warnt ihn unter anderem mit $46 \%$ lifetime risk vor einer Herzattacke und mit genau 0,17\% Wahrscheinlichkeit vor einer Multiplen Sklerose [4].

\section{Wer vor unerwünschten Nebenwirkungen der digitalen Revolution warnt, wird als Relikt einer verflossenen Zeit belächelt.}

Die Selbstmessung der Blutdruck- oder Blutzuckerwerte hat vielen zu besserer Kontrolle verholfen, aber auch ängstliche Personen verunsichert, die sich nachts als Notfälle meldeten. Nun verbreitet sich eine kollektive Neurose der Selbstvermessung, deren positive Effekte übertrieben und deren negative Folgen unterschätzt werden. Immerhin kann man/frau mit 10000 Schritten täglich 40 Rappen Krankenkassenprämie einsparen.

Wer vor unerwünschten Nebenwirkungen der digitalen Revolution warnt, wird als Relikt einer verflossenen Zeit belächelt. Doch sind wir Älteren von Fehlentwicklungen moderner Technik enttäuscht, weil wir ohne Farbfernsehen, Handy, PC und Internet aufgewachsen sind und alle Quantensprünge der Kommunikation und der Information begeistert begrüsst haben Wir tun es heute noch, falls sie - wie immer wieder versprochen - den Menschen und nicht Zahlen und Bezahlungen im Mittelpunkt der Zuwendung belassen

Am Anfang war das Wort - am Ende bleibt die Zahl?
Bildnachweis

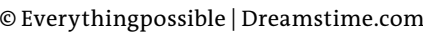
Literatur
1 Lamia P. Ransomware auch im Gesundheitswesen. Schweiz Ärztezeitung .2016;97(49-50):1708-9.
2 Ritter A. Medizin am Wendepunkt. Schweiz Ärztezeitung. 2017;98(5):168-9.
3 Google DeepMind:https://de.wikipedia.org/wiki/Google_Deep- Mind
4 Agus DB, The End of Illness. London: Simon + Schuster; 2012 